\title{
Automation zwischen Insellösung \\ und Logistikkreislauf \\ RFID-gestützte Verknüpfung verschiedener \\ Komponenten als erste Stufe automatisierter \\ Logistikkonzepte in Bibliotheken
}

\section{Olaf Eigenbrodt}

Der Beitrag erläutert anhand der Koppelung von Medienrückgabe, Sortierung und Transport im Jacob- und Wilhelm-Grimm-Zentrum, dass die Verknüpfung verschiedener automatisierter Komponenten ein erster Schritt in Richtung auf Logistikreisläufe in Bibliotheken sein kann. Voraussetzungen und Entwicklung RFID-gestützter automatisierter Kreisläufe werden im zweiten Teil an Beispielen skizziert.

\section{Einleitung}

Für den Einsatz von RFID wurden und werden im Bibliotheksbereich immer neue Anwendungen definiert. Die Verbindung zweier klassischer Probleme der Automatisierung von Routineaufgaben in Bibliotheken - die Identifizierung von Medien zur elektronischen Verbuchung ohne manuelle Eingabe von Identifikationsnummern o. ä. und die Sicherung der Medien gegen unerlaubtes Entfernen aus den Bibliotheksräumen - ist sicherlich für viele Anwender die wesentliche Motivation zur Umstellung auf RFID. Die Technik ermöglichte damit erstmals eine relativ einfache, nutzerfreundliche und zügige Selbstverbuchung von Medien bei geringer Fehleranfälligkeit.

Ähnlich wie die nur unwesentlich ältere aber im Bibliothekswesen schon länger in Einsatz befindlichen Barcodes hat aber die RFID-Technik ein Potential, das wesentlich weiter reicht. Schon früh wurden zusätzliche Anwendungen, wie die automatisierte Inventur bzw. Revision der Bestände oder das Auffinden verstellter Bücher im Bestand beworben. Dabei übernehmen die Bibliotheken in der Regel Anwendungen des Einzelhandels zur Sicherung von Waren sowie zur eindeutigen Identifizierung des individuellen Stücks oder der Warengruppe bei der Transaktion an den Kunden oder der Inventur. Barcode und RFID werden aber schon immer intensiv auch im Bereich der Logistik eingesetzt, wo sie für die effiziente und automatisierte Lagerung genauso eingesetzt werden wie für einen zielgenauen und zeitsparenden Transport. Der reibungslose und genau getaktete Betrieb der Logistik in Handel und produzierendem Gewerbe wäre heute ohne diese Technik nicht mehr vorstellbar. Die eingesetzten Systeme reichen dann von passiven RFID-Transpondern, wie wir sie aus Bibliotheken kennen bis zu aktiven Real-Time-Locating-Systemen (RTLS), wie sie etwa im Containerumschlag verwendet werden. Parallel wird auch immer noch mit Barcodes gearbeitet, wobei die geringere Informationsdichte für die individuelle Verfolgung eines Produkts von der Vorproduktion bis zum Endverbraucher ungeeignet ist, da hier in der Regel nur Chargen verfolgt werden können. 
Auch Bibliotheken müssen diverse Logistikaufgaben lösen, um einwandfrei arbeiten zu können. Im Zuge der wichtigen und nachvollziehbaren Wandlung von einer verwaltungszentrierten zu einer nutzerorientierten Organisation, die unter anderem auch durch den Einsatz von RFID-Technik im Nutzungsbereich unterstützt wird, wird der Aspekt einer reibungslosen Logistik manchmal vergessen. Die führt dazu, dass gerade größere Bibliotheken mit umfangreichen Magazinbeständen im Bereich der Aushebung, des Transports und der Bereitstellung von Materialien, aber natürlich auch bei der Rücknahme, beim Rücktransport ins Magazins und beim Zurückstellen der Materialien, oft noch mit Techniken und Prozessen des 20. oder gar 19. Jahrhunderts arbeiten, obwohl sie im Nutzungsbereich selber längst Technik des 21. Jahrhunderts anbieten.

In diesem Beitrag geht es darum, wie man unterstützt durch den Einsatz von RFID von technisch oft nicht mehr zeitgemäßen Insellösungen zu effizienten Logistikketten kommt, die sich in Bibliotheken meist als Kreisläufe darstellen. Einen Zwischenschritt auf diesem Weg stellen Anlagen wie die im Jacob-und-Wilhelm-Grimm-Zentrum eingesetzte Kombination von Medienrücknahme, -Sortierung und -Transport dar. Deshalb werde ich im Folgenden Vorgeschichte, Konzept und Funktion dieser Anlage im Gesamtkonzept des Hauses vorstellen, bevor ich ein RFID-gestütztes Konzept einer Logistikkette umreiße. In einem Ausblick möchte ich dann noch auf denkbare Logistiklösungen auch für kleinere Bibliotheken eingehen.

\section{Verknüpfung von Komponenten im Jacob-und-Wilhelm-Grimm-Zentrum}

Als eine der ersten Bibliotheken in Deutschland erhielt die Universitätsbibliothek der Humboldt-Universität zu Berlin für ihre neue Zentralbibliothek, das Jacob-und-WilhelmGrimm-Zentrum, eine kombinierte Anlage für Medienrücknahme, -Sortierung und -Transport. Die Verknüpfung dieser drei Komponenten scheint zunächst naheliegend, war aber mit einigen Diskussionen und Herausforderungen verbunden und hatte auch eine Vorgeschichte, auf die ich im Folgenden kurz eingehen möchte.

\section{Von Hasen, Igeln und Selbstverbuchung}

Die Eröffnung des Erwin-Schrödinger-Zentrums auf dem Mathematisch-Naturwissenschaftlichen Campus der Humboldt-Universität zu Berlin in Berlin-Adlershof im Jahre 2003 war ein wesentlicher Entwicklungsschritt der Universitätsbibliothek hin zu einer verstärkten Automatisierung bibliothekarischer Prozesse - allerdings mit unterschiedlichem Erfolg. Damals verzichtete man auf die Einführung von RFID-Technik sowohl im Nutzungsbereich als auch in der Logistik. Im Jahr darauf führte die Universitätsbibliothek der Technischen Universität Berlin RFID zunächst in ihrer Lehrbuchsammlung ein. Im Bereich der Logistik entschied man sich im Erwin-Schrödinger-Zentrum aus Gründen der Gebäudegeometrie- und wegen der damit verbundenen Kostenprognose für eine Kastenförderanlage für ein Fahrerloses Transportsystem (FTS), das unter dem Namen »Hase und Igel« zumindest im deutschen Bibliothekswesen einige Berühmtheit erlangte. Trotz dieser Bekanntheit blieb »Hase und Igel« eine Sonderentwicklung, die als ein Kuriosum des auf ein hochmodernes technisches Erscheinungsbild bedachten Hauses und nicht 


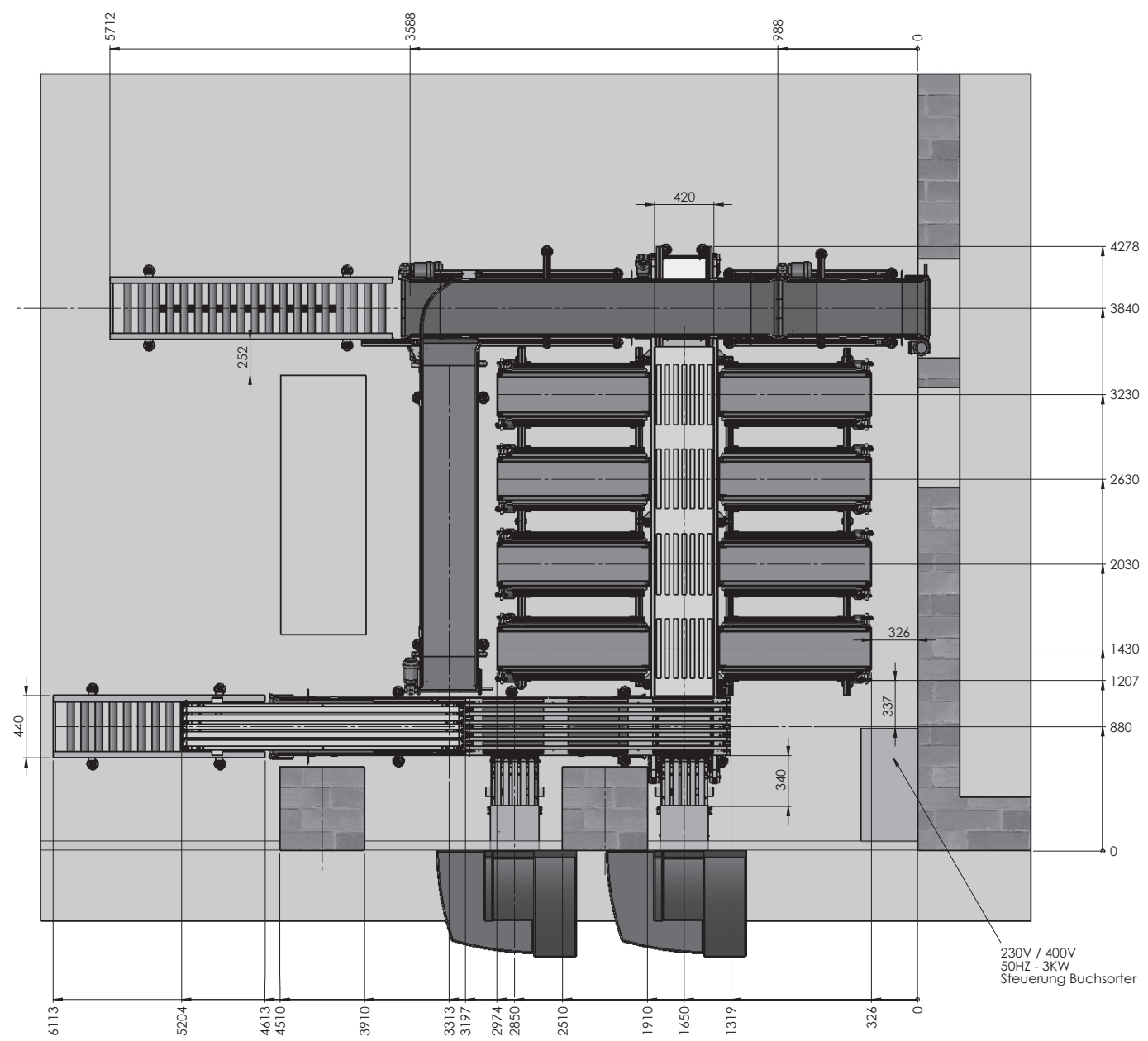

Abb. 1: Aufbau der gekoppelten Anlage im Jacob-und-Wilhelm-Grimm-Zentrum (Zentralbibliothek der Humboldt-Universität zu Berlin)

als eine für die Logistik in Bibliotheken denkbare Entwicklung gesehen wurde. Andreas Richter sieht den Einsatz solcher Systeme in einem eher deskriptiven Beitrag zu Förderund Sortiertechniken folgerichtig auch als problematisch an [vgl. 1, S. 179 f.]. Auf die Möglichkeiten, die ein solches System in der Logistikkette gerade größerer Magazinbibliotheken bieten könnte, geht er leider nicht ein.

Sowohl »Hase und Igel« als auch die im Erwin-Schrödinger-Zentrum eingesetzten Selbstverbuchungsgeräte blieben allerdings Insellösungen. Die Selbstverbuchungsgeräte wurden nach diversen Problemen mit Technik und Bedienerfreundlichkeit der Geräte gar nicht mehr genutzt, während das FTS nach der Beseitigung einiger ,Kinderkrankheiten“ heute seinen geregelten Dienst verrichtet. Durch die erfolgreiche Einführung von RFID im Rahmen des Projekts PROFILE HU verfügt das Erwin-Schrödinger-Zentrum inzwischen über einen Rückgabeautomaten mit angeschlossener Sortieranlage allerdings ohne Koppelung an das FTS. 


\section{Konzept der gekoppelten Anlage im Jacob-und-Wilhelm-Grimm-Zentrum}

Schon bei der Fertigstellung des Erwin-Schrödinger-Zentrums stand fest, dass dies nicht der letzte Neubau der Humboldt-Universität für Bibliothek und Computer- und Medienservice bleiben würde. 2004 wurde der Architekturwettbewerb für das Jacob-undWilhelm-Grimm-Zentrum durchgeführt. Schon in der Ausschreibung für den offenen Realisierungswettbewerb, die die Humboldt-Universität in Zusammenarbeit mit der Senatsverwaltung für Stadtentwicklung erstellte, hatte die Buchtransportanlage sowohl in den Flächenanforderungen als auch in der Beschreibung der Technischen Gebäudeausrüstung eine herausgehobene Stellung [2, S. 58 bzw. 70], auch die Notwendigkeit von Selbstverbuchungsanlagen wurde an dieser Stelle schon erwähnt. Der Einsatz von RFID hingegen wurde zu diesem Zeitpunkt intern zwar diskutiert, war aber noch nicht entschieden. Kostenargumente im Hinblick auf die Gesamtgröße des Bestandes spielten in diesen Diskussionen genauso eine Rolle, wie die damals in den Augen vieler Beteiligter noch nicht ausgereifte Technik.

Im Rahmen der Vorplanung kam man dann relativ schnell zu dem Schluss, dass man die Buchrückgabe nicht nur durch eine Sortieranlage weiter automatisieren, sondern auch an die Transportanlage koppeln sollte. Aufgrund des als geographischer Standort idealen aber für die Gebäudegeometrie schwierigen Baugrundstücks mussten alle Entwürfe zwangsläufig in die Höhe gehen. Der Siegerentwurf des Schweizer Architekten Max Dudler setzte sich über die in der Ausschreibung empfohlene Beachtung der umliegenden Traufhöhen hinweg und erreicht im südlichen Gebäudeteil eine Höhe von 9 Geschossen plus Untergeschoss. Damit war die maximal denkbare Höhenentwicklung allerdings erreicht und man musste mit relativ niedrigen Deckenhöhen arbeiten, um die vorhandenen Höhen optimal auszunutzen. Zum Beispiel wurden alle Leitungen in den Fußboden verlegt, so konnte auf eine Abhangdecke verzichtet werden. Diese konstruktiven Vorgaben und architektonischen Entscheidungen machten eine horizontale Buchtransportanlage konstruktiv und damit auch finanziell zu aufwändig, so dass auf diese verzichtet werden musste. Wegen der geringen Gebäudetiefe wurde dies auch weniger kritisch gesehen, als die Verteilung auf die Gebäudelänge. Stattdessen entschied man sich deshalb für eine vertikale Buchtransportanlage mit zwei Aufzügen.

In diesem Zusammenhang kam dann die Koppelung der mit dem Rücknahmeautomaten verbundenen Sortieranlage mit der Transportanlage ins Gespräch. Während auf der einen Seite ein erheblicher Personalaufwand durch die Verteilung und das Rückstellen der Medien in einem großen Freihandbereich mit einer Kapazität von bis zu 2 Millionen Bänden sicher zu erwarten war und als Organisationsproblem des Freihandbereichs sehr früh thematisiert wurde, wollte man andererseits im Bereich der Sortierung und auch körperlich anstrengenden Umsetzung auf die Kastenförderanlage Personal sparen. Die Idee der Koppelung von Rücknahme, Sortierung und Transport lag damals im Trend und wird auch bei anderen Projekten, die in dieser Zeit geplant und jetzt realisiert wurden eingesetzt.

Konzeptionell stand schnell fest, dass die gesamte Rückgabe der Medien vom Auflegen des Mediums auf das Förderband des Automaten durch den Nutzer bis zur Ankunft des Transportkastens auf der richtigen Etage inklusive einer Benachrichtigung des Personals 
automatisiert werden sollte. Neben der damals also schon gängigen Reduzierung des Sortieraufwands und der erwähnten Umsetzung auf die Förderanlage wurden auch die Zieleingabe der Kästen und die Bestückung der Sortieranlage mit Leerkästen automatisiert. Hier stellten sich für den Bibliotheksbereich völlig neue funktionale Anforderungen.

\section{Funktion der Anlage}

Die drei grundlegenden Komponenten der Anlage - Rücknahmeautomat, Sortieranlage und Kastenförderanlage - sind für sich genommen nicht neu im Bibliotheksbereich, wobei alle drei Lösungen zunächst als Insellösung existierten.

Rücknahmeautomaten können sowohl mit Barcodes als auch mir RFID arbeiten und verbuchen zurückgegebene Medien nach Identifizierung im System der Bibliothek. Hier ist die Schnittstelle zwischen dem Gerät und dem Ausleihmodul der Bibliothekssoftware ein entscheidender Faktor. Rücknahmeautomaten wurden und werden primär dort eingesetzt, wo bis dahin mit Klappenrückgaben für Medien gearbeitet wurde. Der Automat hat für Nutzerinnen und Nutzer den Vorteil, dass zurückgegebene Medien im System verbucht werden. Bibliotheken profitieren ebenfalls von der sofortigen Verbuchung und der relativ höheren Sicherheit vor Vandalismus gerade bei Geräten für einen 24/7-Service, die im Außenbereich angebracht werden. Eine automatisierte Rückgabe über Selbstverbuchungsgeräte war und ist in vielen Bibliotheken schon länger möglich. Hier müssen Kundinnen und Kunden die Medien allerdings selbst auf Bücherwagen ablegen, diese ,verschwinden' nicht in einem Hintergrundarbeitsbereich. In immer mehr Bibliotheken entschließt man sich daher, Rücknahmeautomaten auch im Innenbereich der Bibliothek einzusetzen.

Die Idee, einen Rücknahmeautomaten mit einem Sortierer zu verbinden, liegt eigentlich nahe, wenn es sich um große Bibliotheken oder Bibliothekssysteme mit mehreren Standorten handelt. Allerdings sind auch andere Lösungen denkbar. So werden etwa im Bibliothekssystem Singapurs über Automaten zurückgegebene Medien manuell vorsortiert und dann zur Sortieranlage der Post geliefert, von wo die sortierten Medien dann wieder auf die Standorte verteilt werden. Neben dem entstehenden Transportaufwand hat dieses System auch den Nachteil, dass die Medien manuell vorsortiert und am Zielort noch einmal feinsortiert werden müssen. Allerdings gibt es auch international diverse Beispiele für die gelungene Verbindung von gekoppelter Medienrückgabe und -sortierung. In der Planungsphase der Anlage im Grimm-Zentrum wurden sowohl Anwendungen in Deutschland (Stadtbibliothek München) als auch in Dänemark angesehen. Die Schnittstelle braucht hier zusätzlich Informationen, die bei einem reinen Rückgabeautomaten nicht abgerufen werden müssen: Standort des Exemplars zur richtigen Zuordnung, ggf. Vormerkungen etc. Zudem entschied man sich, zwei Rückgabeautomaten mit der Sortieranlage zu verbinden, was die Komplexität der Sortierung noch erhöht. Die Standardanlagen in diesem Bereich arbeiten in der Regel entweder mit großen Wagen, die über einen Federboden verfügen und so materialschonend relativ hohe Kapazitäten aufweisen (Gerade für den Nacht- oder Sonntagsbetrieb wichtig) oder sie übergeben die Medien direkt an spezielle Bücherwagen, die zwar weniger Kapazität haben, aber direkt weggefahren und in die richtige Abteilung der Bibliothek gebracht werden können. 


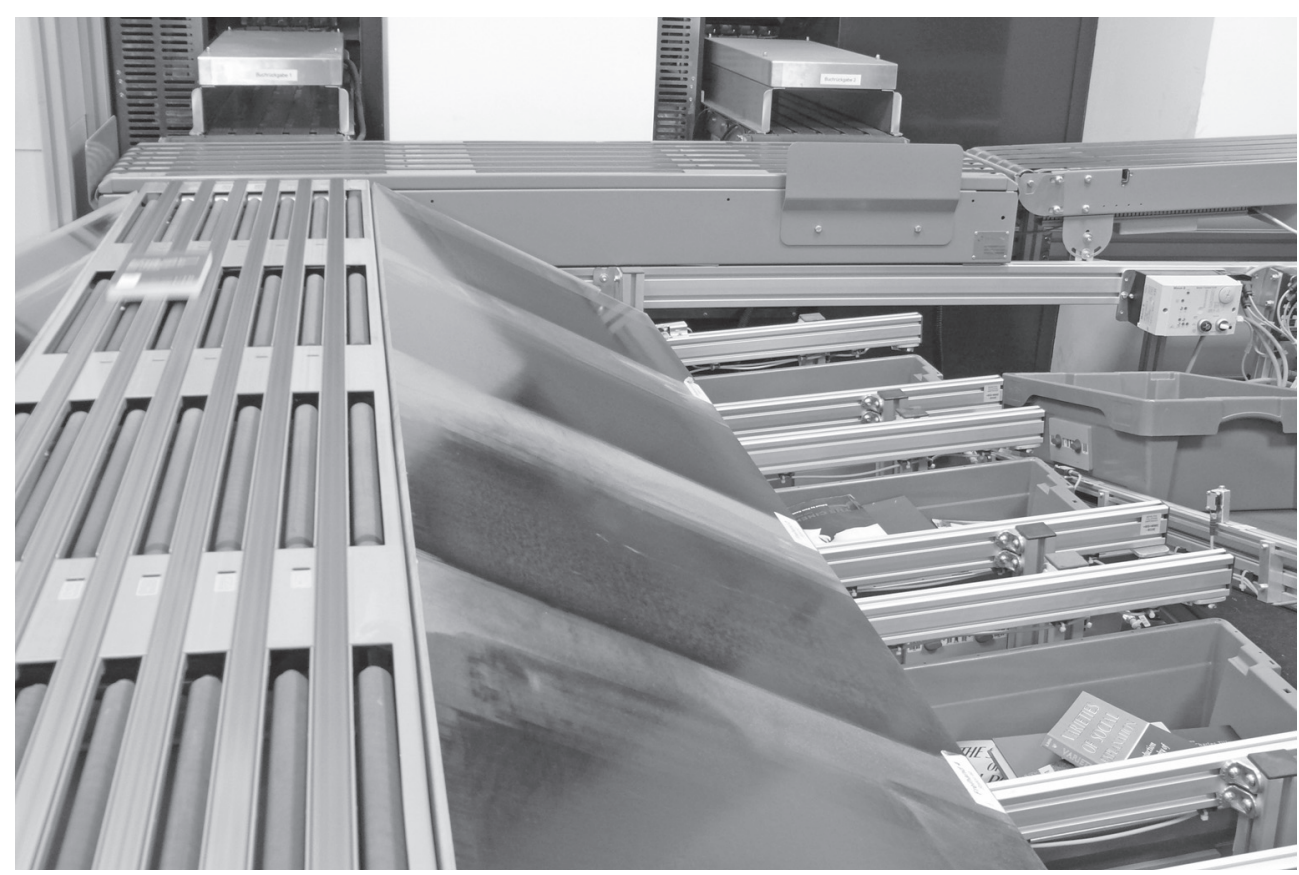

Abb. 2: Die Koppelung von Buchrücknahme, -sortierung und -transport als erster Ansatz eines Logistikkreislaufs

Beide Lösungen haben jedoch Nachteile. So müssen die sortierten Medien entweder in der Nähe der Sortieranlage oder vor Ort noch einmal manuell umgepackt werden. Im Münchener Beispiel geschieht dies sogar zweimal - einmal werden die Medien in die Kästen der Kastenförderanlage sortiert, dann am Zielort noch einmal zum Rückstellen.

Zumindest den ersten Vorgang wollte man sich im Jacob-und-Wilhelm-Grimm-Zentrum durch eine direkte Koppelung an die Kastenförderanlage ersparen. Die Medien werden nicht mehr in spezielle (Bücher-)wagen sortiert, sondern direkt in die Kästen der Förderanlage. Hier entstehen zusätzliche Anforderungen an das System. Zunächst muss eine Schnittstelle zwischen der Sortieranlage und dem Transportsystem geschaffen werden. Zum Zeitpunkt der Planung der Anlage für das Jacob-und-Wilhelm-Grimm-Zentrum gab es keinen Anbieter, der diese Lösung aus einer Hand hätte realisieren können. Alle Bieter im Verfahren arbeiteten mit einem weiteren Partner zusammen. Die Koppelung von Rücknahme und Sortierung war wie erwähnt schon gängig, diese schon vorhandenen Systeme mussten also mit einer Transportanlage verbunden werden. Neben dem Datenaustausch waren auch mechanische Fragen zu lösen: Wie kann man mit Standardtransportkästen arbeiten, ohne die Belastung für die Materialien zu groß werden zu lassen? Was geschieht während der Phase, in der ein voller Kasten abgeholt und gegen einen leeren ausgetauscht wird? Kann die Geschwindigkeit der Transportanlage mit der Kapazität der an zwei Rückgabeautomaten angeschlossenen Sortieranlage mithalten? Gerade die Frage der Bestandserhaltung spielt in einer Wissenschaftlichen Bibliothek mit einer gewissen Archivfunktion quantitativ eine größere Rolle als in einer Öffentlichen Bibliothek, die ihre Materialien in der Regel irgendwann sowieso austauscht. 


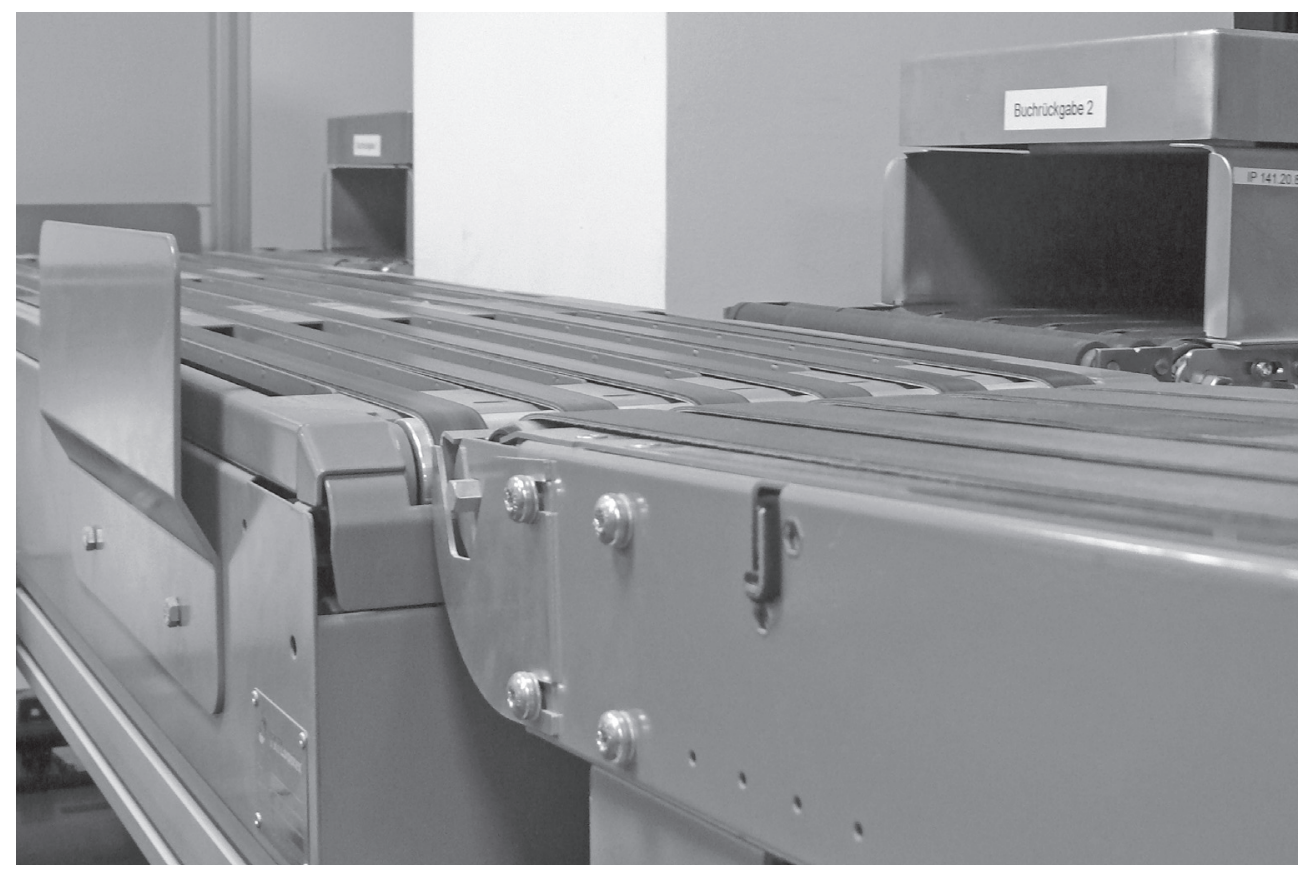

Abb. 3: Zusätzliche manuelle Zuführung von Medien an der Anlage im Jacob-und-Wilhelm-Grimm-Zentrum

Der Planungsprozess erforderte demnach von allen Beteiligten einen hohen Zeit- und Personalaufwand. Neben der Erstellung von Pflichtenheften, der Sammlung zusätzlicher Anforderungen und Detailkonfigurationen mussten auch die Anforderungen an die Schnittstelle zum Aleph-System genau definiert werden. Hinzu kamen Simulationen, Werksbemusterungen von Komponenten und die erwähnten Besuche bereits im Betrieb befindlicher Anlagen.

Entstanden ist eine Anlage mit zwei Rückgabeterminals und acht Sortierungen, die die vollen Kästen über einen Aufzug auf sieben Etagen verteilt und über einen achten Kasten verfügt, der nicht eindeutig zuzuordnende Materialien genauso übernimmt, wie diejenigen Medien, die während des Austausches eines Kastens nicht in diesen zugeordnet werden können. Neben den beiden Rückgabeautomaten verfügt die Anlage noch über eine manuelle Zuführung, die es den Mitarbeiterinnen und Mitarbeitern der Leihstelle ermöglicht, unsortierte Medien von der Sortier- und Transportanlage verteilen zu lassen. Der Füllstand der einzelnen Kästen in der Anlage wird automatisch detektiert und der Kasten bei Bedarf abtransportiert. Die Anlage verfügt über eine eigene Aufzugsstation, der Kasten wird dort übernommen und auf die Etage transportiert, zu der er zugeordnet ist. Hier stehen in der Regel lange Stationen zur Verfügung, die mehrere Kästen aufnehmen können. Sowohl über die VoIP-Telefonanlage als auch über ein Interface auf dem Arbeitsplatzrechner ist der Füllstand der einzelnen Stationen kontrollierbar. Nach der Leerung durch das Bibliothekspersonal werden die Kästen wieder in das System zurückgegeben. Sollte eine Station abends oder am Wochenende volllaufen, können die Kästen in einem Pufferspeicher im Keller zwischengelagert werden, ist die entsprechende 
Station wieder leer, werden die Kästen von dort abgerufen. Ein Leerbehälterspeicher sorgt für eine reibungslose Versorgung mit leeren Kästen. Die Sortieranlage selbst hält immer leere Behälter bereit, um abtransportierte zu ersetzen, wird dort ein Behälter abgerufen, holt sich die Anlage aus dem Leerbehälterspeicher einen neuen.

\section{Herausforderungen und Schwierigkeiten}

Obwohl wie beschrieben von vorn herein der Wunsch nach einer Transportanlage und nach automatisierter Medienverbuchung bestand, wurden Typ und Art der Anlage bewusst offen gelassen, um die Marktentwicklung und den technischen Fortschritt in diesem Bereich abzuwarten. Die Entscheidung zur Koppelung beider Systeme über die Sortieranlage wurde dann zwar zu einem relativ frühen Zeitpunkt getroffen, einige wesentliche Entscheidungen zur Gebäudegeometrie, vor allem auch zu den Verkehrsflächen im Erdgeschoss, waren da aber schon gefallen. In Verbindung mit Verzögerungen bei der Vergabe führte dies dazu, dass zum Zeitpunkt der Auftragserteilung nur noch eine relativ kleine und vor allem auch fest begrenzte Fläche zur Verfügung stand. War die sich dadurch ergebende Kompaktheit der Anlage zunächst eine Herausforderung für die ausführenden Firmen und erst in zweiter Linie ein Problem für die spätere Wartung und Bedienung, so waren die damit verbundene Begrenzung der Sortierungen auf acht und die fehlende Erweiterbarkeit der Anlage ein unmittelbares Planungsproblem. Wie sich schnell herausstellte, wären zur optimalen Vorsortierung der Medien mindestens zwölf Sortierplätze notwendig gewesen. Zusätzlich zu den zu versorgenden Etagen mit ausleihbarem Bestand (UG, EG, 2.-5. OG, 7. OG) hätte man für eine optimale Entlastung des Personals noch Sortierungen für vorgemerkte Medien, Medien anderer Standorte (evtl. mit einer internen Sortierung für einzelne große Standorte oder Fahrtrouten) und nicht eindeutig zuzuordnende Medien gebraucht. Letztere waren zumindest in der Anfangsphase in Folge der Zusammenführung der Bestände aus 13 bis dahin im System selbständig geführten Standorten noch relativ häufig. Hier wäre ein Ansatz zur Weiterführung der automatisierten Logistikkette gewesen, auf den ich später noch eingehen möchte.

Eine besondere Herausforderung stellte die sich relativ spät ergebende Gelegenheit der RFID-Einführung an der Universitätsbibliothek der Humboldt-Universität zu Berlin dar. Trotz der unbefriedigenden Perspektive, die Automatisierung auf der Grundlage der vorhandenen Barcode-Technik voranzubringen, war man aus Kostengründen gezwungen, die im Jacob-und-Wilhelm-Grimm-Zentrum zunächst geplante Einführung von RFID zu verschieben. Durch das vom Europäischen Fond für Regionale Entwicklung (EFRE) geförderte Projekt PROFILE-HU ergab sich die Gelegenheit, die geplante Serviceverbesserung durch Automatisierung auch konsequent umzusetzen und RFID im gesamten Bibliothekssystem der HU einzuführen. So erfreulich diese Entwicklung war, so sehr bedeutete sie auch einen Kraftakt für alle Beteiligten [vgl. dazu und zum Projektverlauf 3]. Insbesondere die Einpassung der bereits geplanten Medienrücknahme, -Sortierungs und -Transportanlage in zwei Projektumgebungen bedeutete einen organisatorischen und technischen Mehraufwand. Hinzu kam, dass man sich im Gegensatz zu den anderen - bedeutend kleineren - Standorten der Universitätsbibliothek im Jacob-und-Wilhelm-Grimm-Zentrum für eine Hybridlösung entschied, da es nicht möglich gewesen 
wäre, bis zur Eröffnung alle Ausleihbestände mit Tags zu versehen [3, S. 592]. Insgesamt ist die Entwicklung zum Einsatz von RFID allerdings absolut positiv zu sehen, auch in Anbetracht künftiger logistischer Herausforderungen gerade in großen und verteilten Bibliothekssystemen.

\section{Automatisierte Logistikkreisläufe im Bibliotheksbereich}

Unter einer Logistikkette versteht man ganz allgemein gesprochen eine Reihe von Prozessen, die notwendig sind, ein Produkt vom Rohstoff bis zum Endverbraucher zu begleiten (Teil der Wertschöpfungskette) im engeren Sinne beschreibt eine Logistikkette den Weg eines Produkts von der Fertigung bis zur Auslieferung an den Kunden. Wichtig sind dabei die Prozessorientierung, also die Auffassung der Logistikkette als einen Prozess, der sich in mehrere Vorgänge aufteilt und die Kundenorientierung, das heißt die Unterordnung des Prozesses unter die Maxime des bestmöglichen Services für die Kundin oder den Kunden. Entsprechend der verbreiteten Auffassung von Logistikketten als Warenketten wurde im Zusammenhang mit RFID-Lösungen hier zumeist über einen denkbaren gemeinsamen Standard von Verlagen bzw. Druckereien und Bibliotheken nachgedacht.

Auch Bibliotheken haben aber spezifische Logistikketten, da sie sich von den meisten Unternehmen dadurch unterscheiden, dass sie ihre Materialien nicht endgültig an die Kundinnen und Kunden abgeben, sondern sie nur verleihen und - in der Regel - nach einiger Zeit zurückerhalten. Die Kette wird hier zu einem Kreislauf. Bott unterscheidet transportlogistische und - unter Rückgriff auf Ewert und Umstätter- informationslogistische Prozesse in Bibliotheken [vgl. 4, S.28]. Im Gegensatz zu Bott würde ich die informationslogistischen Prozesse nicht unbedingt an die transportlogistischen gekoppelt sehen, da die Information nicht immer als Kopie auf einem physischen Träger übergeben wird. Umgekehrt sind aber alle transportlogistischen Aufgaben in der Bibliothek auch informationslogistische und hierin liegt das Potential der RFID in den Logistikkreisläufen von Bibliotheken.

Automatisierung findet in diesem Kreislauf bisher zumeist als Insellösung statt. Schon seit Jahrzehnten wird in großen Bibliotheken der hausinterne Transport von einer Station im Magazin zur Leihstelle und zurück automatisiert, seit einigen Jahren werden zunehmend die Ausleihprozesse durch Selbstverbuchung automatisiert und das oben beschriebene Beispiel zeigt, das man beide Komponenten durch die Sortierung miteinander verbinden kann. Hier kommt dann auch schon - wenn auch nicht notwendigerweise - RFID zum Einsatz. Weitere Anwendungen sind allerdings denkbar, wenn man beginnt, die Logistikkette bzw. den Logistikkreislauf in Bibliotheken als System zu begreifen, das man insgesamt weitgehend automatisieren kann. Im Folgenden möchte ich stichwortartig einige aktuelle Themen im Bezug auf Automatisierung im Logistikkreislauf der Bibliotheken nennen und mögliche Verknüpfungspunkte andeuten.

Dies beginnt mit einfachen Erweiterungen des bestehenden Systems. Ein direkter Anschluss der Transportanlage an Sortierraum und Poststelle hätte eine wesentliche Reduzierung manueller Eingriffe in den Logistikkreislauf mit sich gebracht. So hätte man von dort direkt die richtigen Zielorte für Medien aus anderen Standorten realisieren können, 
die Kisten hätte der Transportdienst nur noch mitnehmen brauchen. Dies ließ sich aufgrund der beschriebenen Gebäudegeometrie nicht realisieren. Wäre die Anlage schon im Gebäudeprogramm etwa über die Definition eines Logistikkreislaufs näher beschrieben worden, hätte sich das sicher in der Entwurfsplanung realisieren lassen. Andere Systeme, deren Einsatzmöglichkeit in Bibliotheken erst in den letzten Jahren wahrgenommen wurde, spielten bei den Planungen im Jacob-und-Wilhelm-Grimm-Zentrum verständlicherweise noch gar keine Rolle.

Mit der zunehmenden Nachfrage nach Arbeitsplätzen und der im Verhältnis dazu abnehmenden Nachfrage nach gedruckten Medien in Wissenschaftlichen Bibliotheken stellt sich die Frage nach dem Flächenverbrauch für die Lagerung der Medien immer dringender. Flächenverbrauch und Unterhaltskosten großer Magazine mit Festplatzlagerung stehen oft in einem problematischen Verhältnis zur Nutzung der Bestände, auch und gerade wenn die Bibliothek einen Archivauftrag zu erfüllen hat und wenig Material aussondern kann. In den Vereinigten Staaten gehen mehr und mehr Wissenschaftliche Bibliotheken und Bibliothekssysteme aus diesem Grund dazu über, Automated Storage and Retrieval Systems (ASRS) einzusetzen, die anstelle eines klassischen Magazins eine digital gesteuerte chaotische Lagerung vornehmen und in ihrer gängigen Form als Vertical-Lift-Moduls (VLM) nicht von Menschen bedient werden müssen. Die Vorteile in der Magazinierung sind hier ein wesentlich geringerer Flächenverbrauch, je nach Einstellung des Systems eine maximale Ausnutzung der zur Verfügung stehenden Kapazität und die Möglichkeit optimaler Lagerungsbedingungen, da die Umweltbedingungen im Magazin menschliche Bedürfnisse nicht berücksichtigen müssen. Darüber hinaus entfallen alle organisatorischen, also verwaltungszentrierten Vorgänge, die in einem Magazin mit Festplatzlagerung notwendig sind. Diese Systeme werden von Bibliothekarinnen und Bibliothekaren oft noch kritisch gesehen, weil ein befürchteter Computerausfall bei der chaotischen Lagerung zum Verlust der Standortnachweise sämtlicher Medien führen könne. Ähnliche Befürchtungen wurden aber auch schon gegen die Abschaffung der Zettelkataloge ins Feld geführt.

Da bestimmte ASRS mit gängigen Transportkisten arbeiten können, ist der Anschluss an ein Transportsystem naheliegend. Ein ASRS Nahbereichs-Magazin kann dann die Kiste mit dem gewünschten Medium im 24/7-Betrieb zeitnah bereitstellen, der Vorgang des Aushebens aus der Kiste müsste weiterhin manuell erfolgen, könnte aber von der Nutzerin bzw. dem Nutzer bei anschließender oder gleichzeitiger Verbuchung per RFID eigenständig erledigt werden. Die Rückgabe könnte sich ähnlich gestalten. Wenn eine Sortiermaschine die Medien nicht nach Standorten, sondern nach Formaten sortiert und die Kästen effizient und möglichst störungsfrei belädt, wäre eine vollautomatische Zuführung zurückgegebener Bücher ins ASRS eine logische Erweiterung des im GrimmZentrum eingesetzten Systems.

RFID-Insellösungen wie intelligente Regale oder Bücherschränke bzw. die Bereitstellung von vorgemerkten Medien lassen sich ebenfalls in einen automatisierten Logistikkreislauf integrieren. Vor allem die relativ einfache Detektion der Medien erlaubt es theoretisch, jedes Medium innerhalb des Logistikkreislaufes ständig zu identifizieren, auch wenn es sich nicht an seinem, von der Festplatzlagerung vorgegebenen Standort befindet. 
Voraussetzung für eine wirtschaftlich vertretbare und effiziente Lösung ist aber immer, den Logistikkreislauf komplett zu analysieren und ggf. neu zu strukturieren. Entscheidend ist dabei zunächst vor der Automatisierung die Optimierung. Unnötige Vorgänge die sich etwa aus der klassischen Magazin- oder Leihstellenorganisation erhalten haben, für einen RFID-gestützten, kundenorientierten Kreislauf aber unnötig sind, sollte man dabei eliminieren. Insgesamt sollte der Kreislauf straffer werden, Bestellungen und Medien durch möglichst wenig Hände gehen, egal ob es sich um Freihandbestände, Magazinbestellungen, Vormerkungen oder Fernleihen handelt. Erst danach sollte die Überlegung stehen, was man RFID-gestützt automatisieren bzw. auf Selbstbedienung umstellen kann.

\section{Fazit}

Die Verknüpfung verschiedener automatisierter Vorgänge innerhalb des Logistikkreislaufs einer Bibliothek, wie sie im Jacob-und-Wilhelm-Grimm-Zentrum mit der Koppelung von Medienrückgabe, -Sortierung und -Transport realisiert wurde, ist ein erster sinnvoller Schritt in Richtung einer weitgehenden Automatisierung. Leider wurden im genannten Beispiel, wie generell im Bibliothekswesen, die einzelnen Vorgänge nicht als ein Logistikkreislauf gesehen, der mit dem Ausheben eines Mediums am Standort (sei es im Freihandbereich oder in einem Magazin) beginnt und mit dem Einstellen desselben Mediums nach einem oder auch mehreren Nutzungsvorgängen wieder endet. Vielmehr schlägt immer wieder die klassische Fokussierung auf die Verwaltung der Medien durch. Will man aber das Potential berührungs- und sichtkontaktfreier Identifikation von Medien, das die RFID-Technik bietet, jenseits von (verknüpften) Insellösungen innerhalb der Kreisläufe nutzen, ist die individuelle Analyse und Optimierung derselben unbedingt notwendig. Hier zeigt sich zunächst, welche Prozesse man gar nicht automatisieren muss, weil sie Rudimente einer Bibliotheksorganisation vor der EDV-Einführung sind oder weil sie nur einer Selbstvergewisserung dienen. In einem zweiten Schritt entwickelt man Verknüpfungen zwischen den definierten Prozessen und erkennt das Automatisierungspotential.

Jedes RFID-Projekt, das sich nicht nur als Insellösung zur Automatisierung bestimmter Vorgänge versteht und jedes (Um-)Bauprojekt im Bibliotheksbereich setzt - unabhängig von der Größe der Bibliothek - eine solche Analyse voraus. Dieser Aspekt ist bisher in der gängigen Literatur zum Bibliotheksbau und -Management aber auch zum RFID-Einsatz in Bibliotheken ein Desiderat und verdient es dringend, eingehender beschrieben und in der Praxis erprobt zu werden. 


\section{Literatur und Internetquellen}

[1] Richter, A. (2009). Förder- und Sortiertechniken. In P. Hauke \& K. U. Werner (Hrsg.), Bibliotheken bauen und ausstatten (S. 175-181). Bad Honnef: BOCK + HERRCHEN.

[2] Humboldt-Universität zu Berlin, Technische Abteilung (Hrsg.). (2004). Jacob und Wilhelm Grimm-Zentrum Berlin Mitte: Zentrale Universitätsbibliothek und Computer- und Medienservice; Offener Realisierungswettbewerb Ausschreibung. Berlin: Senatsverwaltung für Stadtentwicklung.

[3] Berghaus-Sprengel, Anke, Kühne, Tobias. (2009). Das RFID-Projekt an der Bibliothek der Humboldt-Universität zu Berlin - Stand und Perspektiven. Bibliotheksdienst, 43/6, 588-598.

[4] Bott, Anna. (2010). Informationslogistik an einer Hochschulbibliothek unter Berücksichtigung von Erfahrungen aus anderen Industriezweigen: Bachelor Thesis HAW Hamburg. Unveröffentlichtes Manuskript. 